improvement in dyspnoea was reported in 24 of 28 patients $(p<0.001)$, with the cough disappearing in 19 of 21 patients $(p<0.001)$. At 3 months the FEV1 was $>80 \%$ in all 28 patients. The mean exercise capacity was improved in 17 (61\%) patients.

Conclusions The condition of tracheobronchomalacia is a misnomer and we propose the term "Flat Trachea syndrome" and/or "expiratory prolapse of the tracheobronchial posterior membrane". It is a rare but severely debilitating condition which can be diagnosed easily by an awake flexible bronchoscopy and dynamic biphasic inspiratory/expiratory CT. Surgical airway splinting with a PTFE (Teflon) patch considerably improves respiratory symptoms, quality of life and functional status in highly selected patients with this under-diagnosed and under-treated condition.

\section{S57 EVALUATION OF CIRCULATING BIOMARKERS IN LYMPHANGIOLEIOMYOMATOSIS}

doi:10.1136/thx.2010.150938.8

W Y C Chang, L M Magowan, S R Johnson. University of Nottingham, Nottingham, UK

Rationale Lymphangioleiomyomatosis (LAM) is a rare disease of the lungs and lymphatics occurring almost exclusively in women, usually presenting before menopause. It is characterised by progressive cystic destruction of the lung parenchyma, obstruction of lymphatics, airways, and often progressive respiratory failure. Recent diagnostic criteria require lung biopsy in addition to HRCT in the absence of other features such as tuberous sclerosis, renal angiomyolipomas or chylous effusions. The clinical course of LAM varies significantly, and there are no good predictors of clinical progression. Vascular endothelial growth factor D (VEGFD) has been found to be increased in the serum of LAM patients but its role as a biomarker has never been examined prospectively.

We aimed to see if:

1. VEGF-D reduces the need for lung biopsy for diagnosis using a proposed cut off with an estimated test sensitivity for LAM of $86 \%$, specificity of $91 \%$, and a positive likelihood ratio of 9.6.

2. VEGF-D is a useful predictor of severity when correlated with lung function data.

Results Serum samples were taken from 34 LAM patients and 12 healthy controls and a significant difference in VEGF-D levels was seen (Patient median $=768 \mathrm{pg} / \mathrm{ml}, \quad \mathrm{IOR}=417.6-1509$, control median $=329.5, I O R=288.6-489.0, p=0.0082$, Mann-Whitney $U$ test). However, using the proposed cut off of $574 \mathrm{pg} / \mathrm{ml}$ by Young et al (2008), only 1 patient in our cohort with a diagnosis of "probable" LAM would have avoided the need for a lung biopsy to confirm diagnosis. When correlated with lung function, only TLCO demonstrated a statistically significant negative correlation with VEGFD levels $\left(r^{2}=0.2143, p=0.0131\right)$.

Conclusions Though an interesting research tool, the value of VEGFD as a biomarker in LAM has not been clearly demonstrated and there is currently insufficient evidence to advocate its role either to aid in diagnosis or prediction of outcome in LAM.

\section{S58 PULMONARY LANGERHANS' CELL HISTIOCYTOSIS (PLCH): A NEW NATIONAL REGISTER}

doi:10.1136/thx.2010.150938.9

${ }^{1} \mathrm{R}$ H Mason, ${ }^{2} \mathrm{~N}$ M Foley, ${ }^{1} \mathrm{M}$ R Hetzel, ${ }^{1} \mathrm{H}$ Branley, ${ }^{2} \mathrm{~J}$ Suntharalingam. ${ }^{1}$ Oxford Sleep Unit, The Churchill Hospital, Oxford, UK; ${ }^{2}$ Respiratory Department, Royal United Hospital, Bath, UK

Introduction and Objectives PLCH is a rare interstitial lung disease, linked to cigarette smoking and, in some cases associated with respiratory failure and death. Limited UK data have been published and hence little knowledge exists of the diagnostic and treatment practices employed by UK Respiratory physicians. Our study aims to characterise the epidemiological, clinical, histological, radiological and prognostic indicators in a nation wide cohort of patients with PLCH. This study aims to follow the patient journey from point of diagnosis, regardless of the mode of diagnosis, looking at patient demographics and clinical outcomes in the UK population.

Methods UK Respiratory Consultants were contacted to request details for 71 patients with known PLCH, who had previously been registered on the BTS Orphan Lung Disease (BOLD) database. Patients were sent a consent form and questionnaire. Once written consent was obtained, consultants were sent a questionnaire requesting medical information. The patients' GPs have provided current medication and past medical history information.

Results Details on 55 patients were received (including 10 deceased and, 4 lost to follow-up). 17 patients ( 8 males), have returned a completed consent form and questionnaire so far, and were included for analysis. Age at presentation 32.0 years (SD13.7). Presenting symptoms: Shortness of breath $70.5 \%$, pain $47.1 \%$, pneumothorax $11.8 \%$, cough $29.4 \%$ and $5.9 \%$ asymptomatic (diagnosed incidentally). Smoking status: Ex $88.2 \%$, current $6.0 \%$ mean (SD) 22 (27.2) pack years, and 6.0\% never smokers. $23.5 \%$ admitted limited exposure to cannabis. Diagnosis: $82 \%$ patients had had an HRCT scan and $64.7 \%$ an open lung biopsy. Diseasecourse: Symptoms resolved $35.3 \%$, same $41.2 \%$ and $23.5 \%$ had slowly progressed. $47.0 \%$ pneumothorax, $53.0 \%$ have received treatment either chemotherapy 29.4\% (Chlorambucil (2), 2-Chlorodeoxyadenosine (2), Azathioprine (1)) or, an operation for recurrent pneumothorax (23.5\%) 1 patient is on the waiting list for a lung transplant. Patients' opinion of Doctors knowledge of PLCH: High 35.3\%, medium 23.5\%, Med/low or low $41.1 \%$.

Conclusions Although small, this early dataset indicates a high prevalence of smoking in our UK cohort and, that despite advances in CT a high percentage of patients are still diagnosed with an open lung biopsy.

\section{a1-Antitrypsin: what it tells us about COPD S59 A $\alpha$-VAL360: A PLASMA MARKER OF NEUTROPHIL ELASTASE ACTIVITY AND COPD DISEASE SEVERITY}

doi:10.1136/thx.2010.150938.10

${ }^{1} \mathrm{R}$ Carter, ${ }^{2} \mathrm{M}$ J Ungurs, ${ }^{1} \mathrm{R}$ A Stockley. ${ }^{1}$ Queen Elizabeth Hospital Birmingham, Birmingham, UK; ${ }^{2}$ University of Birmingham, Birmingham, UK

In both A1AT deficient and replete subjects, it is widely believed that protease-antiprotease imbalance is central to the pathophysiology of COPD, ${ }^{1}$ although (in the absence of an exacerbation) it is difficult to detect free elastase activity. ${ }^{2}$ This is explained by recent mathematical and in vitro modelling suggesting proteolysis and subsequent enzyme inhibition occur within the immediate microenvironment of the neutrophil. ${ }^{3}$ We have therefore validated a (preinhibition) elastase cleavage product of fibrinogen $\left(\mathrm{A} \alpha-\mathrm{Va}^{360}\right)$ produced in this microenvironment as a potential marker for COPD Methods Pilot study: Plasma A $\alpha-\mathrm{Val}^{360}$ was measured in 68 subjects with a wide range of A1AT levels, and in a further 64 PiZ A1AT deficient subjects, spirometry and plasma $\mathrm{A} \alpha-\mathrm{Val}^{360}$ were measured in the stable state. Subjects with COPD (Normal A1AT levels): 81 subjects were recruited in a primary care setting following an exacerbation associated with sputum production. Trial participants were assessed on day 1 of the exacerbation and in the stable clinical state when full lung function tests and HRCT were also performed. HRCT scans were analysed both densitometrically (voxel index) and visually. 
Results Pilot study: There was an exponential relationship between $\mathrm{A} \alpha-\mathrm{Val}^{360}$ and the A1AT concentration consistent with theoretical modelling and a negative correlation with FEV1 in the PiZ subjects $(\mathrm{r}=-0.321, \mathrm{p}=0.005)$. COPD study (Normal A1AT levels): $\mathrm{A} \alpha-\mathrm{Val} \mathrm{b}^{360}$ was greater in subjects with visible emphysema compared to those without (median 21.77 vs 16.98; $p=0.013$ ) and correlated well with both physiology and densitometry (Abstract S59 Table 1). A $\alpha-\mathrm{Val}^{360}$ was significantly higher in subjects experiencing a purulent versus non-purulent exacerbation (day 1 median 26.29 vs 21.22 ; $p=0.03$ ), and although values fell, the difference persisted even in the stable state $(21.89$ vs $17.01 ; \mathrm{p}=0.002) . \mathrm{A} \alpha-\mathrm{Val}^{360}$ was also higher on day 1 than in the stable state ( 23.72 vs $21.28 ; p=0.005)$ even when stratified into non-purulent ( 21.22 vs $20.00 ; p=0.022)$ or purulent subgroups (26.29 vs $21.83 ; \mathrm{p}=0.043$ ).

Abstract S59 Table 1 The correlation $(r)$ and its significance $(p)$ between stable state plasma $\mathrm{A} \alpha-\mathrm{Val} 360$, physiology and HRCT densitometry

\begin{tabular}{lrr}
\hline & \multicolumn{1}{c}{$\mathbf{r}$} & \multicolumn{1}{c}{$\mathbf{p}$} \\
\hline Body mass index & -0.215 & 0.091 \\
Age & 0.199 & 0.037 \\
FEV1 (\% Predicted) & -0.340 & 0.001 \\
KCO (\% Predicted) & -0.215 & 0.027 \\
TLCO (\% Predicted) & -0.310 & 0.002 \\
Upper Zone Voxel Index & 0.401 & $<0.001$ \\
Lower Zone Voxel Index & 0.340 & 0.001 \\
\hline
\end{tabular}

Conclusion $\mathrm{A} \alpha-\mathrm{Val}^{360}$ correlates well with physiological and radiological markers of COPD disease severity (in subjects with and without A1AT deficiency) and increases during exacerbations (particularly in those with purulent sputum), both supporting the pathophysiological importance of elastase and demonstrating the potential of $\mathrm{A} \alpha-\mathrm{Val}^{360}$ as a valid biomarker in COPD. Further work is required to relate $A \alpha-\mathrm{Val}^{360}$ to longitudinal measures of disease progression.

\section{REFERENCES}

1. Proc Am Thorac Soc 2009;6:524-6.

2. Am Rev Respir Dis 1979:120:1081-6.

3. J Clin Invest 1999;104:337-44.

\section{S60 CIGARETTE SMOKE INDUCED OXIDATION OF $\alpha-1$ ANTITRYPSIN AMPLIFIES THE PULMONARY INFLAMMATORY RESPONSE}

doi:10.1136/thx.2010.150938.11

S Alam, Z Li, R Mahadeva. University of Cambridge, Cambridge, UK

Alpha-1 antitrypsin (AT) is the major elastase inhibitor within the lung. Oxidation of critical methionine residues in AT (Ox-AT) has diminished ability to inhibit neutrophil elastase, which is thought to contribute to the pathogenesis of COPD. Ox-AT may also be proinflammatory. We investigated whether cigarette smoke would promote production of Ox-AT and an exaggerated inflammatory response. Adult female transgenic mice for human M-AT and wild type CBA mice ( $\mathrm{n}=9$ per group) were exposed to cigarette smoke (CS) from 1R3F research grade cigarettes for 5 days and killed 1 day later. Control mice were exposed to air. Ox-AT and inflammatory chemokines were assessed in BALF and lung homogenates (LH) by ELISA and Western blot. Ox-AT was not detected in control M-AT mice nor CS-CBA mice, but was significantly increased in BALF, $72.3 \mathrm{ng} / \mathrm{ml} \quad(\mathrm{SEM} \pm 11.7), \quad \mathrm{p}=0.017$ and $\mathrm{LH}, 1351.3$ ( \pm 111.6$)$ $p=<0.001$ of CS-M-AT mice. This was confirmed on western blot of SDS-PAGE using a monoclonal antibody to Ox-AT. There was a significant increase in BAL polymorphonuclear cells $\left(1.53\left(10^{4}\right)\right.$ $( \pm 0.02)$ vs $\left.0.16\left(10^{4}\right)( \pm 0.04) \mathrm{p}=0.022\right)$ and macrophages $(16.36$ $\left(10^{4}\right)( \pm 0.69)$ vs $\left.10.19\left(10^{4}\right)( \pm 1.94) \mathrm{p}=0.008\right)$ in CS-M-AT mice compared with CS-CBA mice. There was significantly greater MCP1 and $\mathrm{KC}$ in CS-M-AT vs CS-CBA; BALF, MCP-1 $521.35 \mathrm{pg} / \mathrm{ml}$ ( \pm 46.7 ) vs 264.63 ( \pm 17.65$)$, respectively; $p=0.006$, and KC $440.5 \mathrm{pg} /$ $\mathrm{ml}( \pm 53)$ vs $171.4( \pm 17), \mathrm{p}=0.024$. In LH, CS-M-AT MCP-1, 779.6 $( \pm 55)$ vs CS-CBA $368.8( \pm 30)(\mathrm{pg} / \mathrm{ml}) \mathrm{p}=0.003$, and CS-M-AT KC $466.1( \pm 67)$ vs $250.9( \pm 14), p=0.003$. Similarly there was significantly increased NF-kB $(p=0.015)$ and AP-1 $(p=0.015)$ activity in CS-M-AT lungs compared with CS-CBA lungs. These findings demonstrate that oxidation of methionines in AT by oxidants released from cigarette smoke not only reduces the anti-elastase lung protection but converts AT into a pro-inflammatory stimulus. OxAT generated in the airway interacts directly with epithelial cells to release MCP-1 and IL-8, so enhancing lung inflammation. This mechanism could potentially contribute to the abnormal inflammatory response seen in COPD.

\section{S61 CIGARETTE SMOKE PROMOTES POLYMERISATION OF Z a1-ANTITRYPSIN}

doi:10.1136/thx.2010.150938.12

S Alam, Z Li, S Janciauskiene, R Mahadeva. University of Cambridge, Cambridge, UK

Alpha-1 antitrypsin (AT) is an important inhibitor of neutrophil elastase (NE). Z antitrypsin (Glu342Lys) (Z-AT) polymerises within the hepatocyte and the subsequent severe plasma deficiency exposes the lungs to uncontrolled elastolysis and premature emphysema. We have shown that polymeric Z-AT (pZ-AT) are found in emphysematous alveolar walls and are co-localised with neutrophils. pZ-AT does not inhibit NE and are also pro-inflammatory and chemotactic to neutrophils, suggesting a novel role for pZ-AT in Z-AT related emphysema. Cigarette smoking (CS) accelerates decline in lung function in Z-AT homozygotes, but the mechanism involved in this is unknown. We investigated whether CS exposure would induce formation of pZ-AT. Female transgenic mice for human M-AT and Z-AT were exposed to four $1 \mathrm{R} 3 \mathrm{~F}$ research cigarettes daily for 5 days. BALF and perfused lungs were subsequently collected. Concentrations of pAT and oxidised AT were assessed by ELISA and immunoblot. Neutrophil numbers were assessed by quantifying stained cytospins and neutrophil elastase activity of lung homogenates (LH). pAT was undetectable in non-CS Z or CS-M mice. Polymeric AT was markedly increased in BALF and LH in CS-Z mice; BALF CS-Z 141 (146-114) $\mathrm{ng} / \mathrm{ml} ; \mathrm{p}=0.001$ and $\mathrm{LH}, 232.5(241.1-218.6) \mathrm{ng} / \mathrm{lung}$, $\mathrm{p}=0.001$. Immunoblot of BALF demonstrated the classical ladders of pATin CS-Z mice.BALF and LH of CS-Z mice had higher neutrophil numbers compared with CS-M mice; NE LH, CSZ 49(50-45) ng/lung vs CS-M mice 21(25-18); $p<0.001$. Neutrophil numbers in the lung were tightly correlated with polymer concentrations; correlation coefficient, $\mathrm{r}^{2}=0.93 ; \mathrm{p}=<0.001$. Incubation of plasma purified Z-AT with CS extract (CSE) demonstrated that CSE oxidises Z-AT leading to an accelerated rate of polymerisation; CSE $+\mathrm{Z}, \quad 114.4 \mathrm{nM} / \mathrm{h}, \quad \mathrm{Z}$ control 10.3; $\mathrm{p}<0.001$. This was confirmed by the finding that CS-induced polymerisation could be abolished by the antioxidant $\mathrm{N}$-acetyl cysteine CSE+NAC +Z-AT 13.3; $p=0.135$ vs control. In conclusion, acute CS exposure directly promotes polymerisation of Z-AT via oxidation. The production of $\mathrm{pZ}$-AT further reduces the anti-proteinase protection and attracts neutrophils potentially hastening lung damage. These novel findings provide a molecular explanation for the striking 